\title{
Fault Diagnosis of Rolling Bearing Using Wireless Sensor Networks and Convolutional Neural Network
}

\author{
https://doi.org/10.3991/ijoe.v16i11.15959 \\ Liqun Hou ${ }^{(凶)}$, Zijing Li, Huaisheng Qu \\ North China Electric Power University, Baoding, China \\ houliqunencepu.edu.cn
}

\begin{abstract}
Rolling bearings are widely used in modern production equipment. Effective bearing fault diagnosis method will improve the reliability of the machinery and increase its operating efficiency. In this paper, a novel fault diagnosis method based on WSN and CNN has been proposed to fully utilize the strong fault classification capability of $\mathrm{CNN}$ and the inherent merits of WSNs, such as relatively low cost, convenience of installation, and ease of relocation. The feasibility and effectiveness of proposed system are evaluated using the vibration data sets of seven motor operating conditions released by the Case Western Reserve University Bearing Data Center. The experimental results show the fault diagnosis accuracy of the proposed approach can reach $97.6 \%$.
\end{abstract}

Keywords - Fault diagnosis, wireless sensor networks, convolutional neural network, rolling bearing.

\section{Introduction}

Rolling bearings are widely used in modern production equipment and nearly 50 percent mechanical faults are occurred on bearing and related components [1]. For this reason, much attention has been focused on effective bearing fault diagnosis methods [2-4]. It is well known that the structural damage of rolling bearings usually leads to high-frequency and periodic shock on the vibration signal [5-7]. Therefore, identifying such shock signals from the original vibration signal is the key step for fault diagnosis [8]. At present, vibration signals of rolling bearings are generally collected by wired communication. However, the traditional wired network requires a great deal of manpower and material input in wiring, assembling and later maintenance, which may even exceed the price of the sensor itself [9-11].

The emergence of wireless sensor networks (WSNs) solves the above problems in wired networks. In recent years, Internet of things technology gradually attracts people's attention. WSNs, as the carrier of the underlying IoT, develop rapidly as well [12]. WSNs are communications networks composed of a large number of inexpensive wireless microcontrollers that can transmit wireless data in a given area [13]. WSNs are widely used in most fields because of its advantages such as flexible installation position, economical network maintenance, and convenient node replacement [14]. In terms of bearing monitoring, J. Sun et al. [15] proposed a data acquisition and reconstruction 
scheme based on compressed sensing and sparse Bayesian learning algorithm to solve the problem of insufficient power supply of WSN in the industrial environment. This method can reduce the transmission pressure of WSN nodes and improve the life of nodes without affecting the monitoring effect. G. Feng et al. [16] achieve signal acquisition, data processing using fast Fourier transform and Hilbert transform, and feature extraction using envelope spectrum analysis on the end node of WSNs, and then only transmit the fault characteristics to the coordinator node and the PC. Experiments show that this method can reduce data transmission by $95 \%$ compared with direct transmission of raw data. Besides machine condition monitoring and fault diagnosis, WSNs also plays an irreplaceable role in the fields of structure monitoring [17-19], home automation [20], mass transportation [21-22] and security prevention [23].

Deep learning was formed in the 1980s and booming by 2012 with the development of convolutional neural network (CNN) [24-25]. Deep learning has been widely used in object image recognition [26], natural language processing [27], medical diagnosis and treatment [28], infrastructure engineering [29], and other fields due to its excellent feature extraction capability. $\mathrm{CNN}$ as one of the classic deep learning methods has been successfully applied in fault diagnosis. D. Verstraete et al. [30] built a fault diagnosis model based on time-frequency images of vibration signals by utilizing the powerful image recognition capability of CNN. Experiment results show the presented method can get better results with fewer parameters. M. Sadoughi et al. [31] divided feature extraction and CNN fault diagnosis into blocks, first selecting specific data features, and then taking these features as CNN input. This method can effectively solve the problem of simultaneous state monitoring and fault diagnosis of multiple rolling bearings. S. Udmale et al. [32] presented and evaluated a bearing fault diagnosis approach based on kurtogram and deep learning sequence models. D. Belmiloud [33] presented and tested a novel bearing failure prediction method based on wavelet packet decomposition and deep convolutional neural networks. T. Pan et al. [34] proposed and verified a fault diagnosis method for shipborne antenna using 1D-CNN based on multiscale inner product and locally connection feature extraction.

In summary, compared with traditional wired systems WSNs have many inherent merits, while $\mathrm{CNN}$ is a promising deep learning method for bearing fault diagnosis. However, bearing fault diagnosis by combining WSNs and CNN is a relatively unexplored area.

Compared with the above-mentioned bearing fault diagnosis methods, this paper proposes a novel bearing fault diagnosis method based on WSNs and CNN, in which WSNs are used to measure and transmit the bearing vibration signal, while CNN algorithm on a laptop is used for bearing fault diagnosis.

The reminder of this paper is organized as follows. Section II briefly introduces the principle of CNN, while Section III describes the system architecture and implementation methodology. Section IV gives the experimental evaluation of the proposed method. Finally, Section V presents the overall conclusions. 


\section{CNN Principle}

$\mathrm{CNN}$ is generally composed of an input layer, several convolutional layers and pooling layers, a fully connected layer, and an output layer. The convolutional layer and pooling layer can appear alternately more than one times. The convolutional layer is responsible for extracting the feature information from the input signal, while the pooling layer is used to reduce the number of parameters and the computation load. In addition, the fully connected layer can be replaced by the convolutional layer. The structure of the convolutional neural network is shown as Fig. 1.

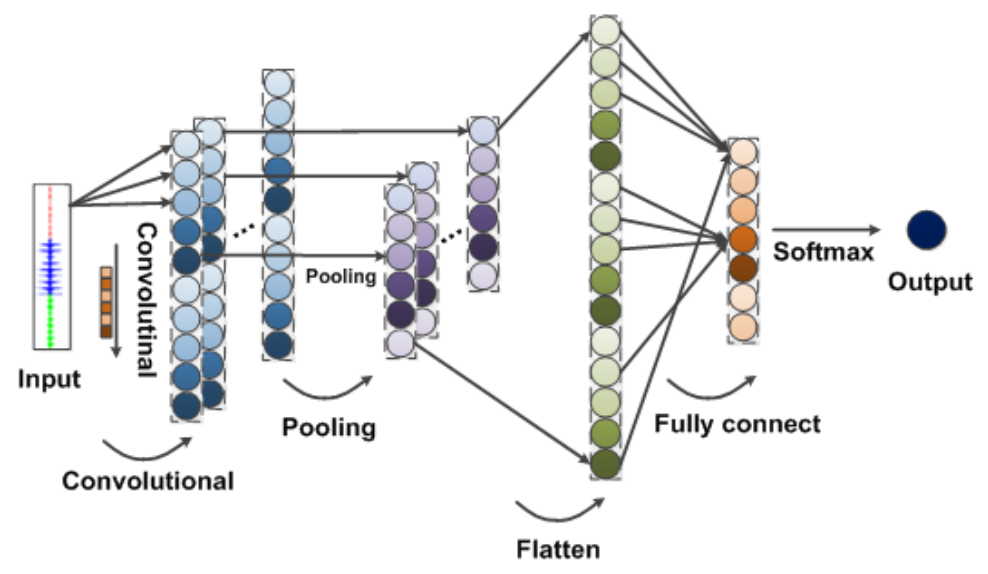

Fig. 1. Convolutional neural network structure diagram

\subsection{Convolutional layer}

The convolutional layer is the core layer of a CNN. It convolves the input signal with different receptive fields to get a new signal. This procedure is named as feature mapping. Different receptive fields can extract different feature maps, which represent various feature information in the original signal. This feature information is the main basis for classification or regression in the fully connected layer. The convolution layer function expression is

$$
H_{j}=f\left(\sum_{i=1}^{p} H_{i}^{\prime} \times W_{i j}+b_{j}\right)
$$

where $H_{j}$ is the $j$-th output feature map of the convolutional layer, $H_{i}^{\prime}$ is the $i$-th input signal of the previous layer, $p$ is the number of receptive fields in the previous layer, $W_{i j}$ represents the weight matrix connecting $H_{i}^{\prime}$ and $H_{j}, b_{j}$ represents the additive bias corresponding to the $j$-th receptive field, $f(\cdot)$ is the excitation function. 


\subsection{Pooling layer}

The main function of the pooling layer is to reduce the size of its input signal, and then reduce the total number of parameters and calculation load of the CNN. By combining the signal in a certain range into a representative value reflecting the most essential characteristics in the range, the pooling layer can effectively reduce the signal size. The pooling area needs to be set according to the actual situation. The pooling layer function is

$$
H_{j}=f\left(\beta_{j} \operatorname{down}\left(H_{j}^{\prime}\right)+b_{j}\right)
$$

Where, $H_{j}$ is the $j$-th output feature map of the current layer, $H_{j}^{\prime}$ is the output feature map of the previous layer, $\operatorname{down}(\cdot)$ represents the pooling rules applied to the pooling layer, $\beta_{j}$ is the $j$-th multiplicative offset of the layer, $b_{j}$ is the $j$-th additive offset of the layer.

\subsection{Fully connected layer}

The fully connected layer, the last layer before the output layer, is generally used to conduct classification or regression. In the modeling of the migration process, the fully connected layer can also play the role of "firewall", especially in the greater difference between the source domain and target domain cases. The connection layer function is given by

$$
y=f\left(W^{T} x+b\right)
$$

Where $y$ is the constant output of the layer, $W$ is the receptive field of the layer, $x$ represents the input signal of the layer, while $b$ represents the additive bias of the layer. In this article, the role of the fully connected layer is to classify bearing faults, so a Softmax function is employed as the activation function for this layer.

\section{System Architecture and Implementation}

This architecture of the proposed bearing fault diagnosis system is illustrated in Fig. 2. The system consists of several WSN end nodes, one WSN coordinator, and a centralized computer. Star topology, IEEE 802.15.4 and ZigBee protocols are used for the WSN. The end nodes and coordinator are used to collect and transmit bearing vibration signal to the centralized computer, while the centralized computer is employed to achieve bearing fault diagnosis based on CNN. This architecture fully utilizes the advantages of WSNs and the computing power of the centralized computer to obtain accurate fault diagnosis result. 


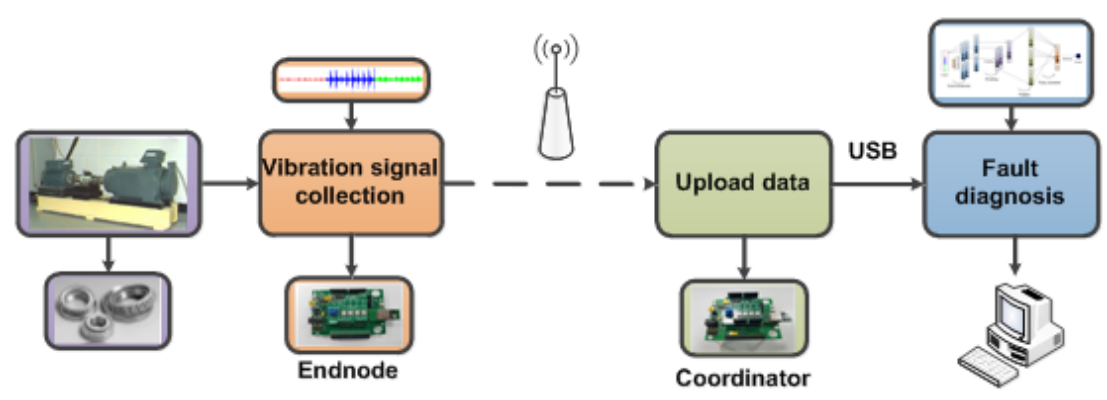

Fig. 2. Fault diagnosis system architecture diagram

\section{Experimental Validation}

\subsection{Experimental setup and procedure}

A series of experiments was conducted to evaluate the proposed bearing fault diagnosis method. The experimental setup used in this research in shown as Fig. 3. One WSN end node transmits the vibration data to the coordinator through ZigBee3.0 protocol, which send the data to the host computer via USB. Then, the developed fault diagnosis APP on the host computer receives and displays vibration data, completes bearing fault diagnosis based on $\mathrm{CNN}$, and gives the final results.

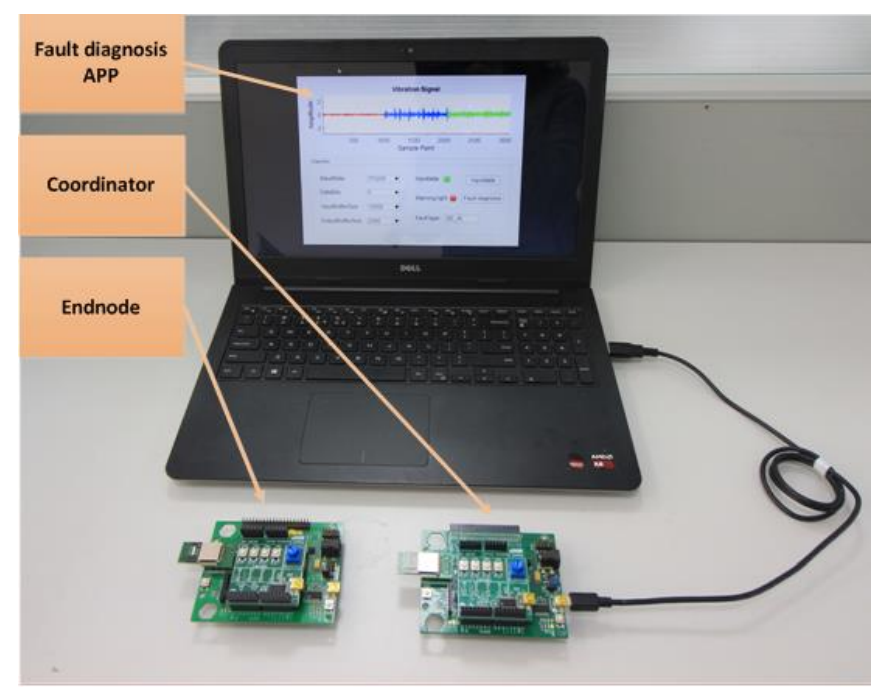

Fig. 3. View of experimental setup

In this project, two NXP JN5169 carrier boards are used as the basic hardware platform for the end node and coordinator. The carrier board includes an NXP JN5169 microcontroller and its peripherals. JN5169 microcontroller supports IEEE 802.15.4 
and ZigBee3.0 protocols, and it integrates a 32-bit RISC architecture with $512 \mathrm{kB}$ Flash, $4 \mathrm{kB}$ EEPROM, and $32 \mathrm{kB}$ RAM, also a fully compliant 2.4 GHz IEEE 802.15.4 transceiver [35]. In addition, JN5169 contains a 128-bit AES security processor to ensure the security during data transmission. The JN5169 is chosen, representing a state-ofthe-art small WSN node suitable for industrial application.

As this paper mainly explores the feasibility of bearing fault diagnosis using WSN and CNN, instead of building up a testbed, this research uses the data set released by the Case Western Reserve University Bearing Data Center [36] and widely used in bearings fault diagnosis fields [4, 37-40]. This data set contains vibration data under fan end fault (FE), driver end fault (FE), and normal state (NOR). Both DE and FE include outer ring fault (OR), inner ring fault (IR) and rotor fault (B). Each fault has three different fault depths, namely 7,14 , and 21 mils.

In this experiment, the bearing vibration data under a rotating speed of $1730 \mathrm{r} \bullet \mathrm{min}-$ 1 and a load of $1 \mathrm{hp}$ are used to evaluate the feasibility of the proposed approaches. The vibration data is from seven bearing operating conditions, namely normal (NOR), driving-end rotor fault (DE-B), driving-end outer ring fault (DE-IR), driving-end outer ring fault (DE-OR), fan-end rotor fault (FE-B), fan-end outer ring fault (FE-IR) and fan-end outer ring fault (FE-OR). 700 sets of vibration data, 100 sets for every operating condition, are employed. Every data set includes $1024 * 3$ sample points, namely the vibrations at base plate, the drive end and the fan end of motor.

The implementation of the proposed bearing fault diagnosis algorithm based on CNN is shown as Fig. 4. It is code using MATLAB on a laptop with Intel $13-32403.4 \mathrm{GHz}$ CPU and 8GB RAM. Part of the vibration data sets are used to train and optimize the $\mathrm{CNN}$. After obtained the optimized parameters of the CNN, these parameters are then embedded in the fault diagnosis APP. Finally, the remained data sets are employed for verifying the effectiveness of the proposed method.

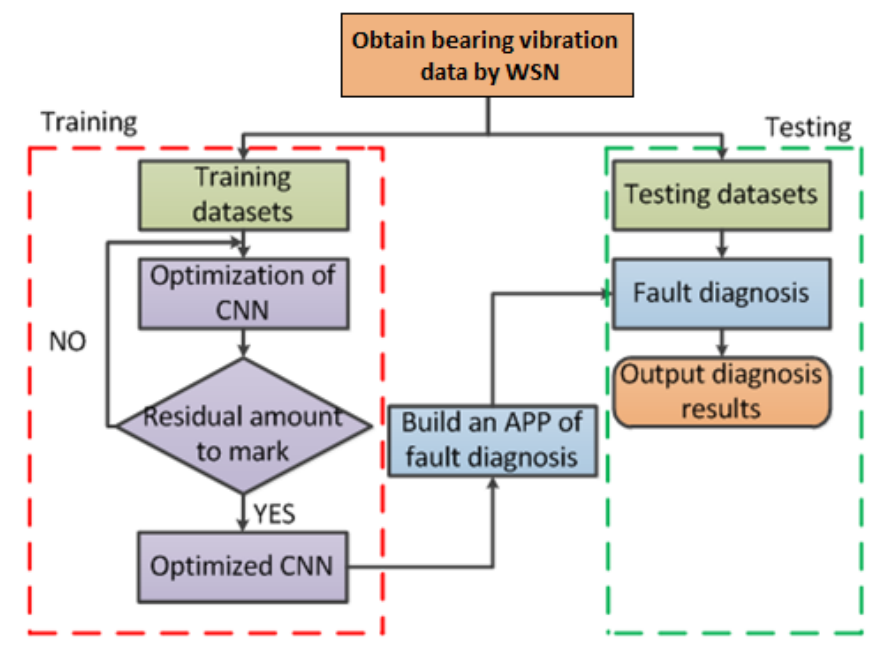

Fig. 4. Fault diagnosis flow chart 
The interface of designed fault diagnosis APP is shown as Fig. 5. As an example, Fig. 5 (a) is for normal condition and Fig. 5(b) is for DE-B condition. The upper portion of the panel is used to display vibration signal curves. The serial port setting including the transmission rate, data bits, input buffer size, and output buffer size is on the lower left corner. The control buttons for "Input Data" and "Fault Diagnosis" and result indication for data transmission and bearing working status are located on the lower right corner.

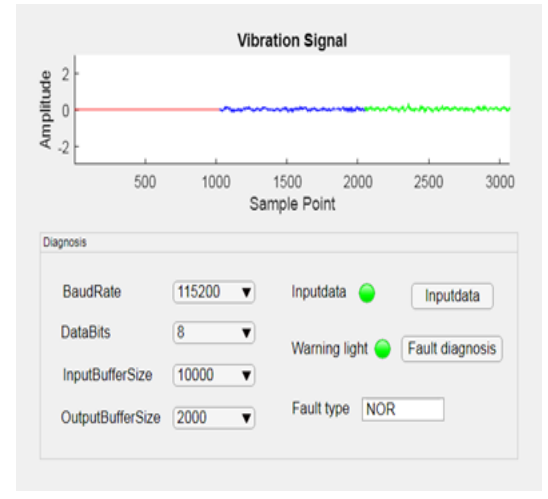

a) normal condition

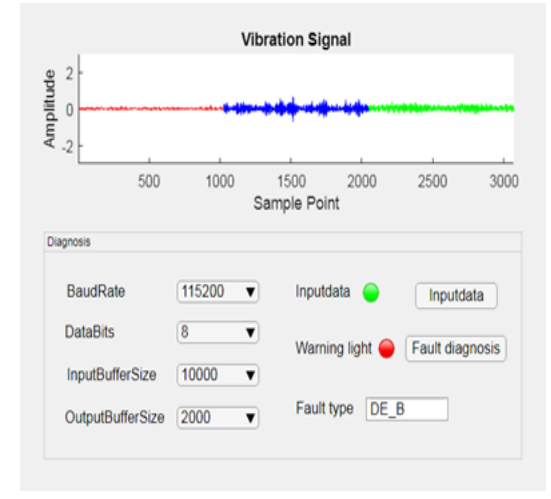

b) DE-B condition

Fig. 5. The interface of fault diagnosis APP.(a) normal condition, (b) DE-B condition

\subsection{Experiment results}

Vibration data transmission: In this experiment, the selected vibration data sets are loaded to the end node in advance to verify the data transmission capability of the proposed system. These data sets are then transmitted to the coordinator wirelessly. When the coordinator receives the data, it sends the data to the host computer by USB immediately. Experimental results show that the proposed bearing fault diagnosis is able to transmit the vibration data wirelessly. Typical vibration signal waveforms for the seven bearing working conditions are given in Fig. 6 . The red, blue, and green curves are the vibration signal measured at the base plate, the drive end and the fan end of motor, respectively. 


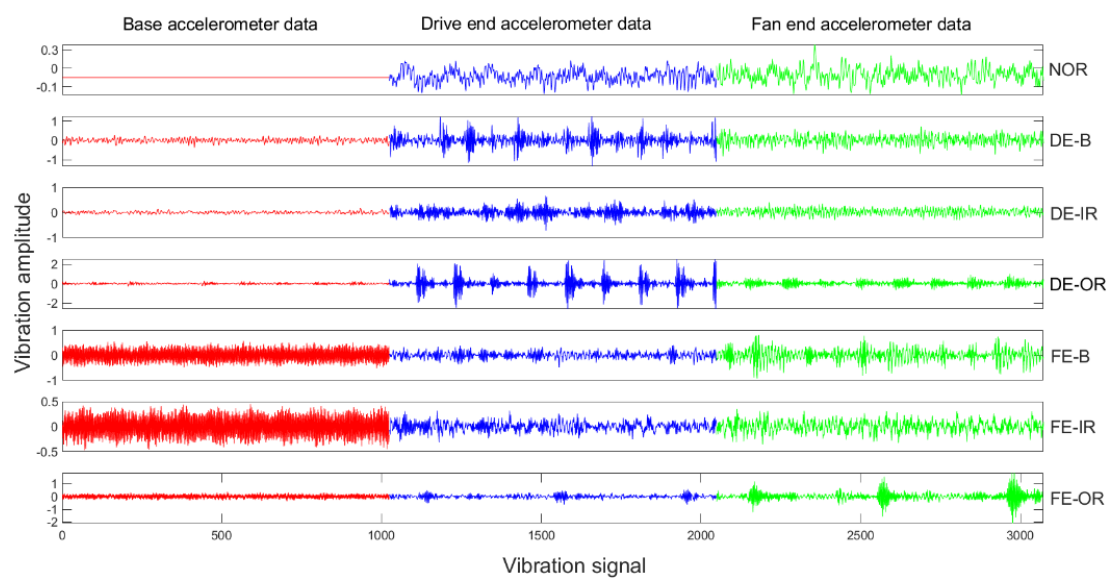

Fig. 6. Vibration signal of rolling bearing

Fault diagnosis accuracy: Considering the accuracy of fault diagnosis and calculation load, the data set used in this experiment consists of $1024 * 3$ sample points, namely three 1024-points vibrations signals that measured at base plate, the drive end and the fan end of motor. Therefore, this paper constructs a basic 2D-CNN consisting of an input layer, an output layer, a convolutional layer, a pooling layer, and a fully connected layer. In order to prevent over-fitting, a dropout layer with a probability of 0.5 is added following the pooling layer during the training process of the $\mathrm{CNN}$ and closed in the fault diagnosis process. The main parameters of convolution layer and pooling layer used in this experiment are given in Table 1. The learning rate, maximum training node, and the mini-batch size of proposed CNN model is set as $0.01,20$, and 5, respectively.

Table 1. Parameters of convolutional layer and pooling layer

\begin{tabular}{|l|c|c|c|}
\hline \multicolumn{1}{|c|}{ Number of layers } & Receptive width & Receptive height & Step size \\
\hline Convolutional layer 1 & 5 & 3 & 1 \\
\hline Pooling layer 1 & 10 & 1 & 1 \\
\hline
\end{tabular}

Shown as Table 2, the output of the $\mathrm{CNN}$ is number 0 to 6 representing the seven bearing working conditions. To achieve accurate fault classification, the Softmax function is used as the output function of the fully connected layer.

Fault type and its corresponding label

\begin{tabular}{|l|c|c|c|c|c|c|c|}
\hline Fault type & NOR & DE-B & DE-IR & DE-OR & FE-B & FE-IR & FE-OR \\
\hline CNN output & 0 & 1 & 2 & 3 & 4 & 5 & 6 \\
\hline
\end{tabular}

Like most neural networks, the fault diagnosis accuracy of CNN depends on the number and quality of the training data set. Therefore, various training-testing data ratio, namely $7: 3,5: 5,3: 7$, and 1:9, are adopted in this experiment to evaluate the fault diagnosis accuracy of the proposed method for the different training data sets size. 
The Classification results of the proposed method with various training-testing data ratio are illustrated by confusion matrix in Fig. 7. It can be seen that when the ratio is 7:3 and 5:5 the fault diagnosis accuracy of the proposed method reaches $100 \%$. When the ratio is 3:7 and 1:9 the fault diagnosis accuracy drops to $99.80 \%$ and $97.6 \%$. For the ratio of 7:3, one FE-B data set is incorrectly classified as FE-IR. However, its location, fan end, is still correct. For the ratio of 1:9, eight DE-OR data set is misdiagnosed as DE-IR, one FE-B data set is misdiagnosed as FE-IR, while six FE-OR data set is misdiagnosed as FE-IR. Although fault diagnosis accuracy significantly decreases for the ratio of 1:9, the classification of fault location is still right.

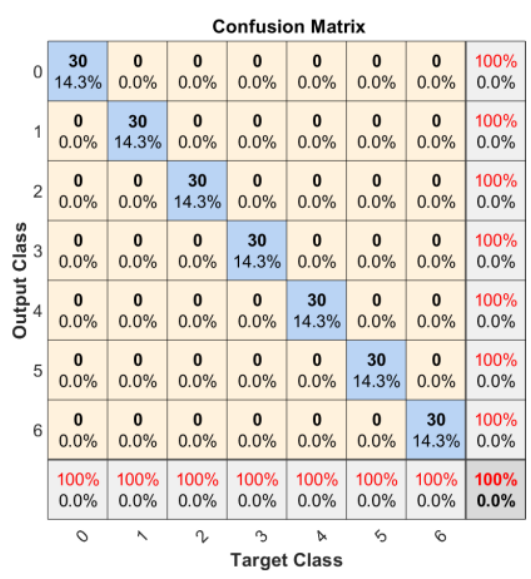

a) $7: 3$

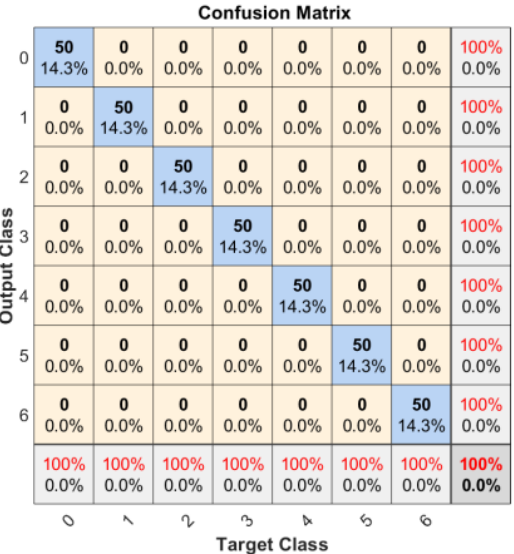

b) $5: 5$

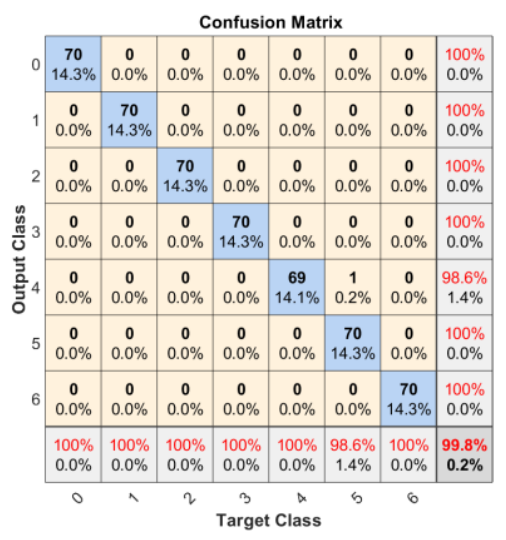

c) $3: 7$

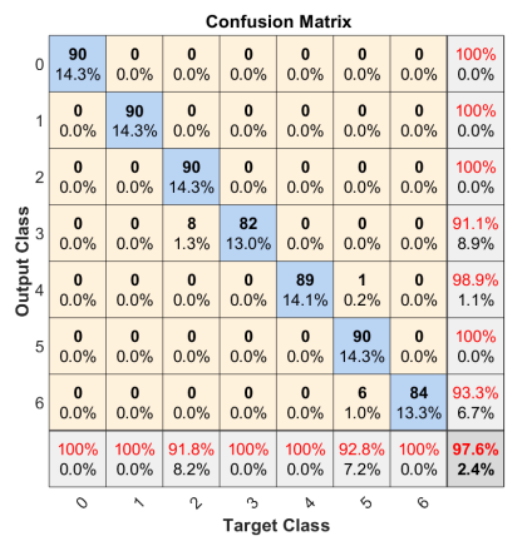

d) 1:9

Fig. 7. Confusion matrix with various training-testing ratios 


\section{Conclusion}

In this paper, a novel fault diagnosis method based on WSN, CNN has been proposed, and evaluated using the vibration data sets of seven motor operating conditions that released by the Case Western Reserve University Bearing Data Center. The experimental results show: (1) the proposed bearing fault diagnosis based on WSNs is able to transmit and display the vibration data smoothly; (2) the fault diagnosis accuracies of the presented method using $\mathrm{CNN}$ reach $100 \%$ when the ratios of training data against testing data are 7:3 and 5:5, while the accuracies reach $99.8 \%$ and $97.6 \%$ for the ratios of $3: 7$ and $1: 9$.

\section{Acknowledgement}

The Natural Science Foundation of Hebei Province, China (Grant No. supported this work F2016502104), the Scientific Research Foundation for the Returned Overseas Chinese Scholars by The Ministry of Education of the People's Republic of China, and the Fundamental Research Funds for the Central Universities of China.

\section{$7 \quad$ References}

[1] M. M. M. Islam, J. Kim, "Automated bearing fault diagnosis scheme using 2D representation of wavelet packet transform and deep convolutional neural network," Computers in Industry, vol. 106, Apr., pp.142-153, 2019. https://doi.org/10.1016/j.compind.2019.01.008

[2] Z. Chen, S. Deng, X. Chen, et al., "Deep neural networks-based rolling bearing fault diagnosis," Microelectronics Reliability, vol. 75, Aug., pp. 327-333,2017. https://doi.org/10. 1016/j.microrel.2017.03.006

[3] S. Tyagi, S. K. Panigrahi, "An improved envelope detection method using particle swarm optimisation for rolling element bearing fault diagnosis," Journal of Computational Design and Engineering, vol. 4, no. 4, Oct., pp. 305-317, 2017. https://doi.org/10.1016/j.jcde.20 17.05 .002

[4] S. M. Y.Nikravesh, H. Rezaie, M. Kilpatrik, and H. Taheri, "Intelligent fault diagnosis of bearings based on energy levels in frequency bands using wavelet and support vector machines (SVM)," Journal of Manufacturing and Materials Processing, vol.3, no.1, Jan., pp. 11, 2019. https://doi.org/10.3390/jmmp3010011

[5] Y. G. Lei, Intelligent fault diagnosis and remaining useful life prediction of rotating machinery. Saint Louis, MO: Elsevier Science, 2016. https://doi.org/10.1016/b978-0-12811534-3.00006-8

[6] P. K. Kankar, S. C. Sharma, S. P. Harsha, "Rolling element bearing fault diagnosis using wavelet transform," Neurocomputing, vol. 74, no. 10, May, pp.1638-1645, 2011. https:// doi.org/10.1016/j.neucom.2011.01.021

[7] X. Wang, Y. Zi, Z. He, "Multi-wavelet denoising with improved neighboring coefficients for application on rolling bearing fault diagnosis," Mechanical Systems and Signal Processing, vol. 25, no. 1, Jan., pp. 285-304, 2011. https://doi.org/10.1016/j.ymssp.2010.03. $\underline{010}$ 
[8] B. Chen, B. Shen, F. Chen, et al., "Fault diagnosis method based on integration of RSSD and wavelet transform to rolling bearing," Measurement, vol. 131, Jan., pp. 400-411, 2019. https://doi.org/10.1016/j.measurement.2018.07.043

[9] B. F. Spencer, H. Jo, K. A. Mechitov, et al., "Recent advances in wireless smart sensors for multi-scale monitoring and control of civil infrastructure," Journal of Civil Structural Health Monitoring, vol. 6, no. 1, Feb.,pp 17-41. https://doi.org/10.1007/s13349-015-0111-1

[10] A. H. Alavi, H. Hasni, N. Lajnef N, et al., "Damage detection using self-powered wireless sensor data: an evolutionary approach," Measurement, vol. 82, Mar., pp. 254-283, 2016. https://doi.org/10.1016/j.measurement.2015.12.020

[11] B. Qin, L. Zhang, H. Yin, et al., "Multiple feature vectors basvectors-basedsification for WSN integrated bearing of rolling mill," Journal of Control Science and Engineering, vol. 2018, Apr., pp.1-11, 2018. https://doi.org/10.1155/2018/3041591

[12] L. Hou, J. Hao, Y. Ma, and N. Bergmann, "IWSNs with on-sensor data processing for energy efficient machine fault diagnosis," International Journal of Online Engineering, vol. 15, no. 8, pp. 42-61, 2019. https://doi.org/10.3991/ijoe.v15i08.10314

[13] H. Chen, C. Wu, W. Huang, et al., "Design and application of system with dual-control of water and electricity based on wireless sensor network and video recognition technology," International Journal of Distributed Sensor Networks, vol. 14, no. 9, Sept., pp. 15501477 18795951, 2018. https://doi.org/10.1177/1550147718795951

[14] G. Fan, J. Li, H. Hao, "Lost data recovery for structural health monitoring based on convolutional neural networks," Structural Control and Health Monitoring, vol. 26, no. 10, Aug., pp. e2433, 2019. https://doi.org/10.1002/stc.2433

[15] J. Sun, Y. Yu, J. Wen, "Compressed-sensing reconstruction based on block sparse bayesian learning in bearing-condition monitoring," Sensors, vol. 17, no. 6, June, pp. 1454, 2017. https://doi.org/10.3390/s17061454

[16] G. Feng, J. Gu, D. Zhen, et al., "Implementation of envelope analysis on a wireless condition monitoring system for bearing fault diagnosis," International Journal of Automation and Computing, vol. 12, no. 1, Feb., pp. 14-24, 2015. https://doi.org/10.1007/s11633-014-0862$\underline{\mathrm{x}}$

[17] O. Avci, O. Abdeljaber, S. Kiranyaz, et al., "Wireless and real-time structural damage detection: A novel decentralized method for wireless sensor networks," Journal of Sound and Vibration, vol. 424, June, pp. 158-172, 2018. https://doi.org/10.1016/j.jsv.2018.03.008

[18] J. Meech, C. Crabtree, Z. Rácz, "Star type wireless sensor network for future distributed structural health monitoring applications," Inventions, vol. 4, no. 1, Jan., pp. 6, 2019. https://doi.org/10.3390/inventions4010006

[19] H. Salehi, S. Das, S. Biswas, et al., "Data mining methodology employing artificial intelligence and a probabilistic approach for energy-efficient structural health monitoring with noisy and delayed signals," Expert Systems with Applications, vol. 135, Nov., pp.259-272, 2019. https://doi.org/10.1016/j.eswa.2019.05.051

[20] N. K. Suryadevara, S. C. Mukhopadhyay, S. D. T. Kelly, et al., "WSN-based smart sensors and actuator for power management in intelligent buildings," IEEE/ASME transactions on mechatronics, vol. 20, no. 2, Apr., pp. 564-571, 2014. https://doi.org/10.1109/tmech.2014. $\underline{2301716}$

[21] M. Wolf, J. Hofbauer, M. Rudolph, "Diagnostics using self-sufficient wireless sensor network for a condition-based maintenance strategy strategy for tram bearing diagnostics," 2016 13th International Multi-Conference on Systems, Signals \& Devices (SSD), 2016, pp.518-522. https://doi.org/10.1109/ssd.2016.7473698

[22] S. A. Putra, B. R. Trilaksono, M. Riyansyah, et al., "Intelligent sensing in multiagentbased wireless sensor network for bridge condition monitoring system," IEEE Internet of 
Things Journal, vol. 6, no. 3, June, pp. 5397-5410, 2019. https://doi.org/10.1109/jiot.2019. 2901796

[23] W. Khalid, A. Sattar, M. A. Qureshi, et al., "A smart wireless sensor network node for fire detection," Turkish Journal of Electrical Engineering \& Computer Sciences, vol. 27, no. 4, pp. 2541-2556, 2019. https://doi.org/10.3906/elk-1812-81

[24] C. Le, B. E. Boser, J. S. Denke, et al., "Handwritten digit recognition with a back-propagation network," Proceedings of the 2nd International Conference on Neural Information Processing Systems'02, 1990, pp. 396-404.

[25] A. Krizhevsky, I. Sutskever, G. E. Hinto, "Imagenet classification with deep convolutional neural networks," Proceedings of the 25th International Conference on Neural Information Processing Systems'01, 2012, pp. 1097-1105.

[26] P. N. Druzhkov, V. D. Kustikov, "A survey of deep learning methods and software tools for image classification and object detection," Pattern Recognition and Image Analysis, vol.26, no. 1, Jan., pp. 9-15, 2016. https://doi.org/10.1134/s1054661816010065

[27] R. Sarikaya, G. E. Hinton, A. Deoras, “Application of deep belief networks for natural language understanding," IEEE/ACM Transactions on Audio, Speech and Language Processing (TASLP), vol. 22, no. 4, Apr., pp. 778-784, 2014. https://doi.org/10.1109/taslp. 2014.2303296

[28] Á. S. Hervella, J. Rouco, J. Novo, et al., "Deep multi-instance heatmap regression for the detection of retinal vessel crossings and bifurcations in eye fundus images," Computer Methods and Programs in Biomedicine, vol. 186, Apr., pp. 105201, 2020. https://doi.org/ 10.1016/j.cmpb.2019.105201

[29] Y. Liu, S. Hou. "Rockburst prediction based on particle swarm optimization and machine learning algorithm," International Conference on Inforatmion technology in Geo-Engineering, 2019, pp. 292-303. https://doi.org/10.1007/978-3-030-32029-4_25

[30] D. Verstraete, A. Ferrada, E. L. Droguett, et al., "Deep learning enabled fault diagnosis using time-frequency image analysis of rolling element bearings," Shock and Vibration, vol. 2017,Oct., pp. 1-17, 2017. https://doi.org/10.1155/2017/5067651

[31] M. Sadoughi, C. Hu. "Physics-based convolutional neural network for fault diagnosis of rolling element bearings," IEEE Sensors Journal, vol, 19, no. 11, June, pp. 4181-4192, 2019. https://doi.org/10.1109/jsen.2019.2898634

[32] S. S. Udmale, S. K. Singh, S. G. Bhirud. "A bearing data analysis based on kurtogram and deep learning sequence models," Measurement, vol. 145, Oct., pp.665-677, 2019. https:// doi.org/10.1016/j.measurement.2019.05.039

[33] D. Belmiloud, T. Benkedjouh, M. Lachi, et al., "Deep convolutional neural networks for Bearings failure predictionand temperature correlation," Journal of Vibroengineering, vol. 20, no. 8, Dec., pp. 2878-2891, 2018. https://doi.org/10.21595/jve.2018.19637

[34] T. Pan, J. Chen, Z. Zhou, et al., "A novel deep learning network via multiscale inner product with locally connected feature extraction for intelligent fault detection," IEEE Transac tions on Industrial Informatics, vol. 9, no. 15, Sept., pp. 5119-5128,2019. https://doi.org/ 10.1109/tii.2019.2896665

[35] "Support Resources for JN516x MCUs," [Online]. Available: https://www.nxp.com.cn/ products/wireless/zigbee/support-resources-for-jn516x-mcus:SUPPORT-RESOURCESJN516X-MCUS. [Accessed: Oct. 30, 2019].

[36] "Case Western Reserve University Bearing Data Center," [Online]. Available: http://csegroups.case.edu/bearingdatacenter/home. [Accessed Nov. 28, 2019].

[37] F. Ding, M. Qiu, X. Chen, "A fault diagnosis method of bearing using energy spread spectrum and genetic algorithm," Journal of Vibroengineering, vol. 21, no. 6, Sept., pp.16131621, 2019. https://doi.org/10.21595/jve.2018.19961 
[38] S. Kass, A. Raad, J. Antoni, "Self-running bearing diagnosis based on scalar indicator using fast order frequency spectral coherence," Measurement, vol. 138, May, pp. 467-484, 2019. https://doi.org/10.1016/j.measurement.2019.02.046

[39] N. Enshaei, F. Naderkhan, "Application of deep learning for fault diagnostic in induction machine's bearings," 2019 IEEE International Conference on Prognostics and Health Management (ICPHM), 2019, pp. 1-7. https://doi.org/10.1109/icphm.2019.8819421

[40] V. H. Nguyen, T. D. Hoang, V. T. Thai, et al., "Big vibration data diagnosis of bearing fault base on feature representation of autoencoder and optimal LSSVM-CRO classifier model," 2019 International Conference on System Science and Engineering (ICSSE), 2019, pp. 557-563. https://doi.org/10.1109/icsse.2019.8823332

\section{Authors}

Liqun Hou (corresponding author) is an Associate Professor in the School of Control and Computer Engineering, North China Electric Power University, Baoding 071003, China. E-mail: houliqun@ncepu.edu.cn

Zijing Li is currently working toward the M. Eng. degree at North China Electric Power University, Baoding 071003, China. E-mail: lizijing9602@163.com

Huaisheng Qu is currently working toward the M. Eng. degree at North China Electric Power University, Baoding 071003, China. E-mail: quhuaisheng@163.com

Article submitted 2020-06-03. Resubmitted 2020-07-14. Final acceptance 2020-07-16. Final version published as submitted by the authors. 